\title{
Residual Stress and Its Effect on Yielding in SiC/Ti Plate*
}

\author{
Fenghua ZHOU**, Ryosaku HASHIMOTO***, Akinori OGAWA*** and Yasushi SOFUE*** \\ Thermal residual stress (TRS) created in metal matrix composite influences the mechan- \\ ical properties of composites. In this paper, we present the TRS-modulated tensile properties \\ of continuous $\mathrm{SiC}$ fiber reinforced titanium $(\mathrm{SiC} / \mathrm{Ti})$ composite. The magnitude and distri- \\ bution of TRS inside $\mathrm{SiC} / \mathrm{Ti}$ were evaluated by using a simple 1-D beam model considering \\ fiber-matrix structure and then a full 3-D FEM model. The bending of the SiC/Ti specimen \\ resulting from the asymmetrical fiber placements was used as a measure to verify the per- \\ tinence of the mechanical models. A 3-D FEM simulation accounting all TRS information \\ gives a good prediction of the composite's tensile properties.
}

Key Words: $\quad \mathrm{SiC} / \mathrm{Ti}$ Composite, Thermal Residual Stress, Micromechanical Model, Tensile Property

\section{Introduction}

Metal matrix composites (MMCs) are good candidates in aerospace structures. A good summation of the early investigations on the thermo-mechanical properties of MMCs can be found in Ref.(1). Many analytic or numerical studies have been conducted to investigate the fiber/matrix interactions in order to explain or predict the composite's behavior ${ }^{(2)-(5)}$. In our group, continuous silicon carbide fiber reinforced titanium alloy $(\mathrm{SiC} / \mathrm{Ti})$ has been investigated for possible applications in aeroengines $^{(6)}$. Tensile tests of $\mathrm{SiC} / \mathrm{Ti}$ showed that the thermal residual stress (TRS) inside the material significantly influences its properties ${ }^{(7),(8)}$. The TRS effect must be considered in order to correctly predict the material's properties $^{(8)}$.

TRS in $\mathrm{SiC} / \mathrm{Ti}$ composite is introduced during the cooling process of fabrication. We can evaluate the magnitude and distribution of TRS using a thermal-mechanical model. One unanswered question is, how accurate is this thermal stress calculated from the model? In this paper we will present a measure to verify the accuracy of the mechanical model. In the following sections we describe the two TRS-related phenomena during a test: the specimen bending and the early yielding. Then we establish an

* Received 7th October, 2005 (No. 05-4248)

** Department of Mechanical Engineering, Johns Hopkins University, 3400 North Charles Street, Baltimore, Maryland 21218, U.S.A. E-mail: fzhou@ @ jhu.edu

*** Aviation Program Group, Japan Aerospace Exploration Agency, 7-44-1 Jindaiji Higashi-machi Chofu-shi, Tokyo 182-8522, Japan analytical model with fiber-matrix structural details. We firstly solve the mechanical problem using simple beam theory to get closed expressions of specimen bending and uniaxial thermal stress distribution. Then we conduct 3D FEM simulation to calculate the triaxial TRS components within the composite. The specimen bending curvatures calculated by both analyses are close, and agree with the actual measurements. This confirms the accuracy of the model. We further extend the 3D FEM simulation to obtain the composite's nonlinear stress-strain curve and compare it with the experimental one.

\section{Experimental Results of $\mathrm{SiC} / \mathrm{Ti}$}

Four plies of unidirectional $\mathrm{SiC}$ fiber pre-forms (Textron SCS- $6^{\mathrm{TM}}$ ) were aligned and stacked with seven plies of titanium alloy foils $\left(\mathrm{NKK} \mathrm{SP700}{ }^{\mathrm{TM}}\right)$ in $\left(\mathrm{Ti}_{2} /(\mathrm{SiC} / \mathrm{Ti})_{4} / \mathrm{Ti}\right)$ sequence, and were hot-pressed at $775^{\circ} \mathrm{C}$. The manufactured $\mathrm{SiC} / \mathrm{Ti}$ composite plate has an average thickness $1.1 \mathrm{~mm}$. This plate was cut along the fiber direction, and furnished in both surfaces to form four $1 \mathrm{~mm}$-thick tensile specimens. Side views of the specimens are shown in Fig. 1 (a). Because of the asymmetrical material removal all specimens bent. The bending curves of the specimens were almost parabolic. The centers of the $(L) 120 \mathrm{~mm}$ long specimens numbered $\mathrm{N} 3, \mathrm{~N} 2$, $\mathrm{N} 1$ and $\mathrm{N} 4$ bent by $\left(w_{\max }\right) 2.0,3.1,3.6$ and $4.6 \mathrm{~mm}$, respectively. These values are converted into the bending curvature $\kappa: \kappa=8 w_{\max } / L^{2}$ giving values of $1.11 \times 10^{-3}$, $1.72 \times 10^{-3}, 2.00 \times 10^{-3}$ and $2.56 \times 10^{-3} \mathrm{~mm}^{-1}$, respectively. The tensile tests were conducted at room temperature. Experimental engineering $\sigma-\varepsilon$ curves are shown in Fig. 7. Fractographs of the specimens are shown in Fig. 1 (b). The 


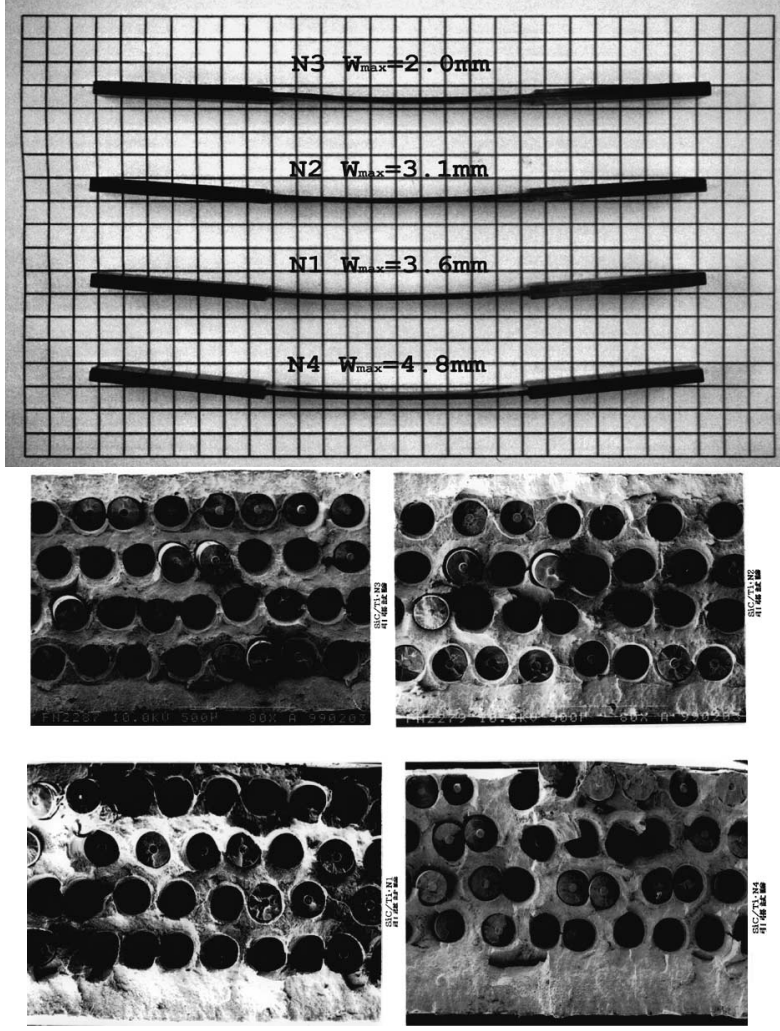

Fig. 1 (a) Side view of specimen before tests; (b) cross-sections of SiC/Ti Specimen: N3, N2, N1, N4

fiber volume ratio $V^{f}$, measured from the cross-sectional pictures, is roughly $31 \%$ for all four specimens. Existence of TRS is observed from following phenomena:

1. Before test, the specimen bent due to the asymmetric placements of fibers within matrix. The more asymmetric the fiber placement was, the larger the specimen bent.

2. The SiC fiber is considered as elastic-brittle and titanium matrix elastic-perfect plastic. Their properties are listed in Table 1. According to the "law of mixtures", the composite will be bi-linear elastic-plastic. The yielding point corresponds to matrix yield; and the failure point corresponds to fiber breakage, as:

$$
\begin{aligned}
& \text { Elasticity: } \begin{array}{l}
E_{L}=E^{f} V^{f}+E^{m}\left(1-V^{f}\right) \\
v_{L T}=v^{f} V^{f}+v^{m}\left(1-V^{f}\right)
\end{array} \\
& \varepsilon_{y}=\varepsilon_{y}^{m}=\frac{\sigma_{y}^{m}}{E^{m}} \\
& \text { Plasticity: } \sigma_{y}=\left(\frac{E^{f}}{E^{m}} V^{f}+1-V^{f}\right) \sigma_{y}^{m} \\
& E_{p}=E^{f} V^{f}
\end{aligned}
$$

$$
\text { Failure : } \sigma_{s}=\sigma_{s}^{f} V^{f}+\sigma_{y}^{m}\left(1-V^{f}\right)
$$

where $E$ is Young's modulus and $v$ the Poisson ratio. The superscript $\left({ }^{f}\right)$ and $\left({ }^{m}\right)$ refer to fiber and matrix, respectively. One can estimate by Eq. (2) that the composite should have the yield strain $0.83 \%$ and the yield stress $1750 \mathrm{MPa}$. However, by experiments, the average yield
Table 1 Mechanical/thermal parameters of the constituents

\begin{tabular}{|c|c|c|c|}
\hline \multicolumn{2}{|c|}{} & $\begin{array}{c}\text { SiC Fiber } \\
\left(\mathrm{SCS}-6^{\mathrm{TM}}\right)\end{array}$ & $\begin{array}{c}\text { Ti Matrix } \\
\left(\mathrm{SP} 700^{\mathrm{TM}}\right)\end{array}$ \\
\hline \multirow{3}{*}{ Elasticity } & $\mathrm{E}(\mathrm{GPa})$ & 400 & 127 \\
\cline { 2 - 4 } & $v$ & 0.18 & 0.27 \\
\hline \multirow{3}{*}{ Plasticity } & $\sigma_{\mathrm{y}}(\mathrm{MPa}) / \varepsilon_{\mathrm{y}}$ & - & $1050 / 0.0083$ \\
\cline { 2 - 4 } & Yielding Law & - & Von Mises \\
\cline { 2 - 4 } & Work Hardening & - & N/A \\
\hline \multicolumn{2}{|c|}{ Failure Strength $\sigma_{\mathrm{s}}(\mathrm{MPa})$} & 3450 & - \\
\hline \multicolumn{2}{|c|}{$\mathrm{CTE}\left(10^{-6} 1 /{ }^{\circ} \mathrm{C}\right)$} & 4.5 & 10.3 \\
\hline
\end{tabular}

strain was $0.6 \%$, lower than expected; and the experimental average yield stress was $1100 \mathrm{MPa}$, much less than the predicted value. Thus the "law of mixtures" failed to give an accurate estimation of the composite's behavior. This phenomenon is linked to the TRS existence. Actually the matrix (titanium alloy) was pre-tensioned by residual stress, and therefore would yield in an earlier stage of tensile loading ${ }^{(8),(9)}$.

\section{Fiber-Matrix Model and Solutions}

To predict the macroscopic mechanical behavior of the composite, it is critical to evaluate the TRS property inside the material. The TRS was originated in the final cooling process of material's fabrication, when temperature decreases from HIP temperature $\left(T_{0}\right)$ down to room temperature $\left(T=25^{\circ} \mathrm{C}\right)$. As the coefficient of thermal expansion (CTE) of metallic matrix is larger than that of ceramic fiber, in the cooling process tensile stress is introduced in matrix and compressive stress in fibers. In the current model, we assume that the properties of both fiber and matrix are temperature independent, as listed in Table 1. This assumption is appropriate when the parameters listed above are regarded as the average values across $\left(T \sim T_{0}\right)$ temperature range.

Various analytical models have been proposed to evaluate the TRS state inside unidirectional fiber reinforced composite. The simplest one is the 1-D bar model. This model assumes that the composite is in 1dimensional stress state, i.e., only normal stress component in fiber direction, $\sigma_{0}$, exists, and both fiber and matrix deform by the same normal axial strain $\varepsilon_{0}$. Given $\Delta T=T-T_{0}$ temperature change, the thermal strain along the axis of composite and the axial thermal stress of each constituents are:

$$
\left\{\begin{array}{l}
\varepsilon_{0}^{f}=\varepsilon_{0}^{m}=\frac{E^{f} \alpha^{f} V^{f}+E^{m} \alpha^{m}\left(1-V^{f}\right)}{E_{L}} \Delta T \\
\sigma_{0}^{m}=\frac{V^{f} E^{m} E^{f}\left(\alpha^{m}-\alpha^{f}\right)}{E_{L}} \Delta T \\
\sigma_{0}^{f}=\frac{\left(1-V^{f}\right) E^{m} E^{f}\left(\alpha^{m}-\alpha^{f}\right)}{E_{L}} \Delta T
\end{array}\right.
$$

where $E_{L}$ is the equivalent longitude modulus given in Eq. (1), and $\alpha$ the CTE parameter.

This model, nevertheless, does not provide information on stress distribution and specimen curving. The dif- 


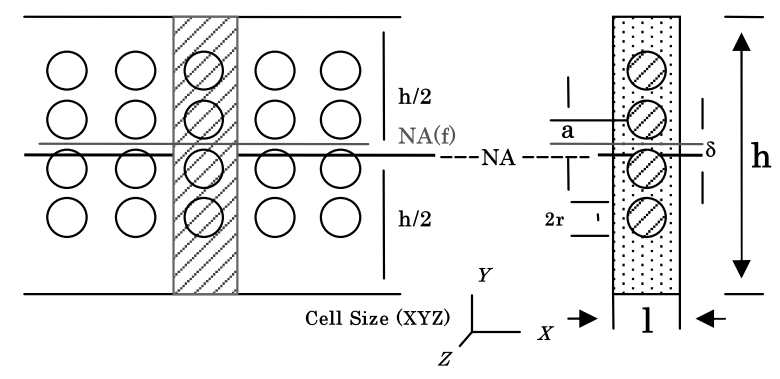

Fig. 2 Micromechanical model for analysis

ferences of current SiC-Ti structure (Fig. 2) from the bar model are: (a) In the $X$-direction the fibers are close to each other so that their interactions are not negligible; (b) In the $Y$-direction the fibers are subject to the influences of the upper and lower free surfaces. Therefore the stress state in the transverse plane ( $X Y$ plane) is complicated. To evaluate the residual thermal stress, a mechanical model is established referring to the realistic fiber-matrix structure. The model is illustrated in Fig. 2. The specimen thickness (h) is $1 \mathrm{~mm}$; within the transverse section four layers $\mathrm{SiC}$ fibers are distributed at an interval (2a) $0.196 \mathrm{~mm}$; in each layer, fibers are distributed at an interval $(l) 0.198 \mathrm{~mm}$; each SiC fiber has the same diameter $(2 r) 0.14 \mathrm{~mm}$. The fiber volume ratio $V^{f}$ is $31 \%$.

A parameter $\delta$, defined as the distance between the centerline of the fiber column and the neutral axis (NA) of the specimen is introduced to represent the asymmetry of fiber placement in the specimen. When fibers are symmetrically placed $\delta$ equals to $0 . \delta$ increases as the fibers deviates toward the upper surface of the plate. The largest value of $\delta$ is $\delta_{0}=136 \mu \mathrm{m}$ when fiber column touches the surface of specimen. A relative fiber asymmetry $\Delta$ is defined as: $\Delta=\delta / \delta_{0}$.

\subsection{A $1 \mathrm{D}$ beam analysis of thermal bending}

The above 1-D bar model is modified as a beam model. We assume uniaxial stress state in composite constituents but allow the specimen to bend. Under thermal load $\Delta T=T-T_{0}$, the plate deformed with a normal strain $\varepsilon_{0}$ at the neutral axis and a bending curvature $\kappa$ in fiber axis. Strain distribution in a cross-section of the specimen is:

$$
\varepsilon(y)=\varepsilon_{0}+\kappa y
$$

where $y$ is the distance from the point being considered to the neutral axis of specimen section. The normal stress in fiber and matrix are:

$$
\left\{\begin{array}{l}
\sigma^{f}(y)=E^{f}\left(\varepsilon_{0}+\kappa y-\alpha^{f} \Delta T\right) \\
\sigma^{m}(y)=E^{m}\left(\varepsilon_{0}+\kappa y-\alpha^{m} \Delta T\right)
\end{array}\right.
$$

As the specimen is free, the resultant force and moment in cross-section is balanced:

$$
\left\{\begin{array}{l}
N=\int_{S_{f}} \sigma^{f}(y) d s+\int_{S_{m}} \sigma^{m}(y) d s=0 \\
M=\int_{S_{f}} \sigma^{f}(y) y d s+\int_{S_{m}} \sigma^{m}(y) y d s=0
\end{array}\right.
$$

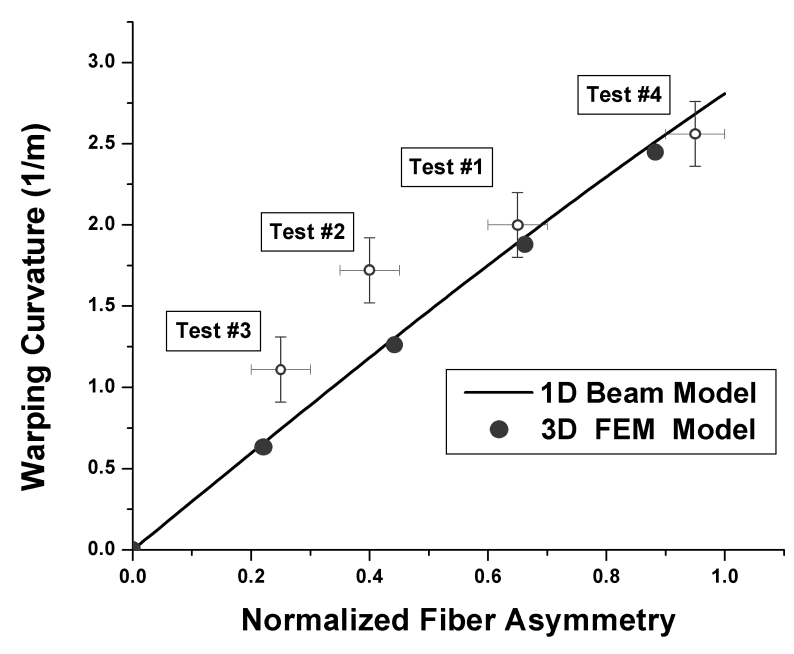

Fig. 3 Specimen curvature $\kappa$ versus fiber asymmetry $\Delta$

Substitute Eq. (6) into Eq. (7), and with some mathematical manipulations, we have following explicit expressions for the thermal axial/bending deformation $\left(\varepsilon_{0} \text { and } \kappa:\right)^{(10)}$ :

$$
\begin{aligned}
& \left\{\begin{array}{c}
\varepsilon_{0}=\frac{\left(C_{2} D_{0}-C_{1} D_{1}\right) \Delta T}{C_{0} C_{2}-C_{1}^{2}} \\
\kappa=\frac{\left(C_{0} D_{1}-C_{1} D_{0}\right) \Delta T}{C_{0} C_{2}-C_{1}^{2}}
\end{array}\right. \\
& \left\{\begin{array}{l}
C_{0}=\left(E^{f}-E^{m}\right) V^{f}+E^{m} \\
D_{0}=\left(E^{f} \alpha^{f}-E^{m} \alpha^{m}\right) V^{f}+E^{m} \alpha^{m} \\
C_{1}=\left(E^{f}-E^{m}\right) V^{f} \delta \\
D_{1}=\left(E^{f} \alpha^{f}-E^{m} \alpha^{m}\right) V^{f} \delta \\
C_{2}=\left(E^{f}-E^{m}\right) V^{f}\left(\frac{r^{2}}{4}+5 a^{2}+\delta^{2}\right)+E^{m} \frac{h^{2}}{12} \\
D_{2}=\left(E^{f} \alpha^{f}-E^{m} \alpha^{m}\right) V^{f}\left(\frac{r^{2}}{4}+5 a^{2}+\delta^{2}\right)+E^{m} \alpha^{m} \frac{h^{2}}{12}
\end{array}\right.
\end{aligned}
$$

Equation (8) applies for any fiber distribution within the plate. Normal stress in the fiber and matrix are calculated when Eq. (8) is substituted into Eq. (6).

A special case is that fibers were placed symmetrically across the neutral axis, i.e., $\delta=0$. In this case $C_{1}=D_{1}=0$. Then the beam results $(5,6,8)$ are reduced to the bar results Eq. (4) as expected.

The first equation in Eq. (8) gives a analytical relationship between $\kappa$ and $\delta$. Figure 3 plots the curve of $\kappa$ verses the normalized asymmetry $\Delta=\delta / \delta_{0}$ and compares it with the experimental data. The $1 \mathrm{D}$ beam model gives results that are reasonably close to the experimental measurements. This is considered an indirect verification on the pertinence of our model.

\subsection{D FEM simulations}

To account for the TRS triaxiality that is important to the material's yielding behavior, we conduct a full 3D FEM analysis to evaluate TRS components in all directions. Because each fiber extends endlessly in the $Z$ direction (fiber axis), and the fiber columns are distributed 


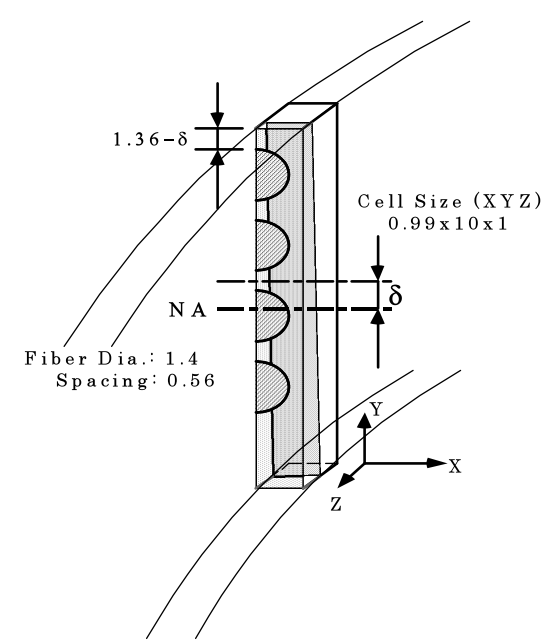

(a)

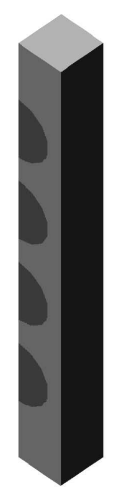

Model 0

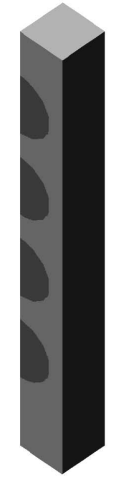

Model 1

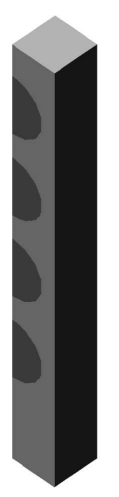

Model 2

(b)

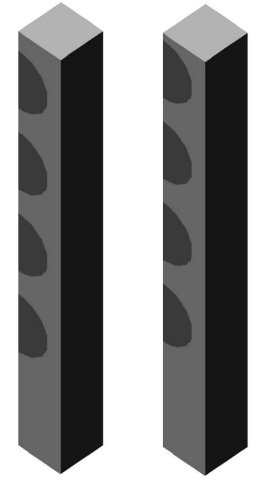

Model 3 Model 4

Fig. 4 (a) A unit cell for 3D FEM simulation; (b) models with different fiber asymmetry

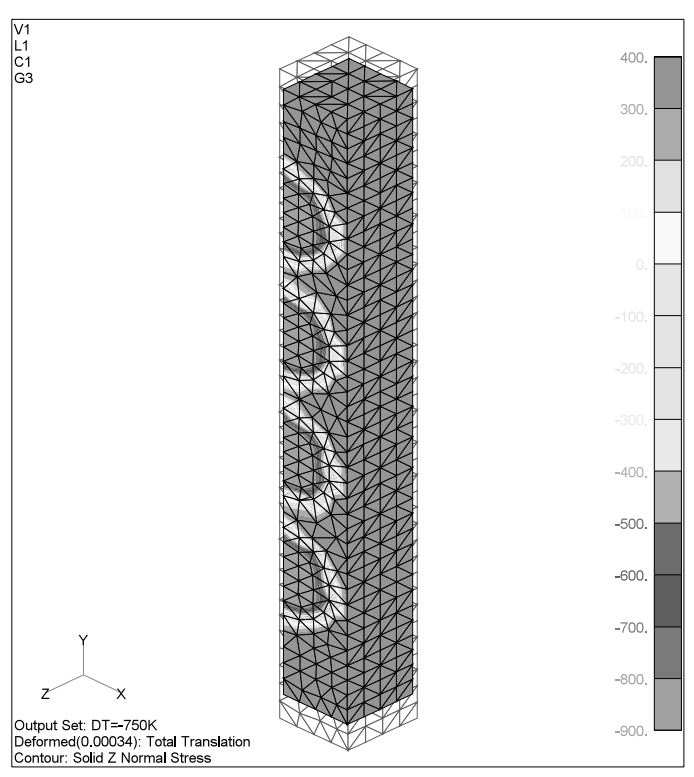

(a) periodically in $X$-direction, a unit cell, shown in Fig. 4 (a), is cut out for FEM analysis. This cell has the height $(h)$ $1.0 \mathrm{~mm}$ in $Y$-direction, depth $(d) 0.1 \mathrm{~mm}$ in $Z$-direction and width $(l / 2) 0.099 \mathrm{~mm}$ in $X$-direction. Five models, Model 0 through 4 with $\delta$ values 0, 30, 60, 90 and $120 \mu \mathrm{m}$ were simulated (Fig. 4 (b)). In each model cyclic or symmetrical boundary conditions are applied. Table 2 details these BCs. The fiber-matrix interface is assumed to be perfect. The structural FEM software NASTRAN is used for calculations. Geometric nonlinearity is included in the FEM model.

The cell is subjected to temperature decrease from HIP temperature $\left(775^{\circ} \mathrm{C}\right)$ to room temperature $\left(25^{\circ} \mathrm{C}\right)$. The thermal deformation and the axial thermal stress $\left(\sigma_{z z}\right)$ distribution within the specimen are shown in Fig. 5(a) and (b), corresponding to symmetric and asymmetric cases $(\delta=0 \mu \mathrm{m}$ and $120 \mu \mathrm{m})$, respectively. In general when subjected to a temperature decrease, both the fiber and the matrix contract. However, the contractions of the constituents are different. This contraction difference results in compressive stress in $\mathrm{SiC}$ fiber which has a smaller CTE, and tensile stress in surrounding titanium matrix.

Table 2 Boundary conditions of the representative cell

\begin{tabular}{|c|c|c|}
\hline Surfaces & Features & Math Expression \\
\hline$x=0$ & X- symmetric & $u_{x}=0$ \\
\hline$x=0.099$ & Remains plane & Rigid plan with free $u_{x}$ \\
\hline$z=0$ & Z- symmetric & $u_{z}=\mathbf{0}$ \\
\hline$z=0.1$ & Remains plane & Rigid plane with free $u_{z}$ \\
\hline $\mathrm{y}=0$ or 1 & Free & No constraint applied \\
\hline
\end{tabular}

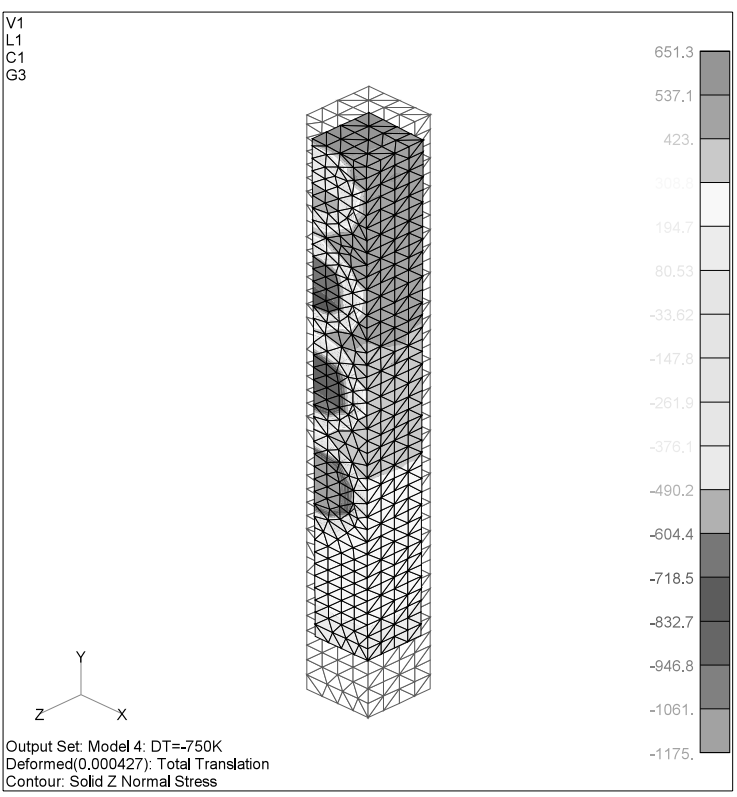

(b)

Fig. 5 Thermal deformation and normal TRS distribution in (a) Model 0 case; and (b) Model 4 case 


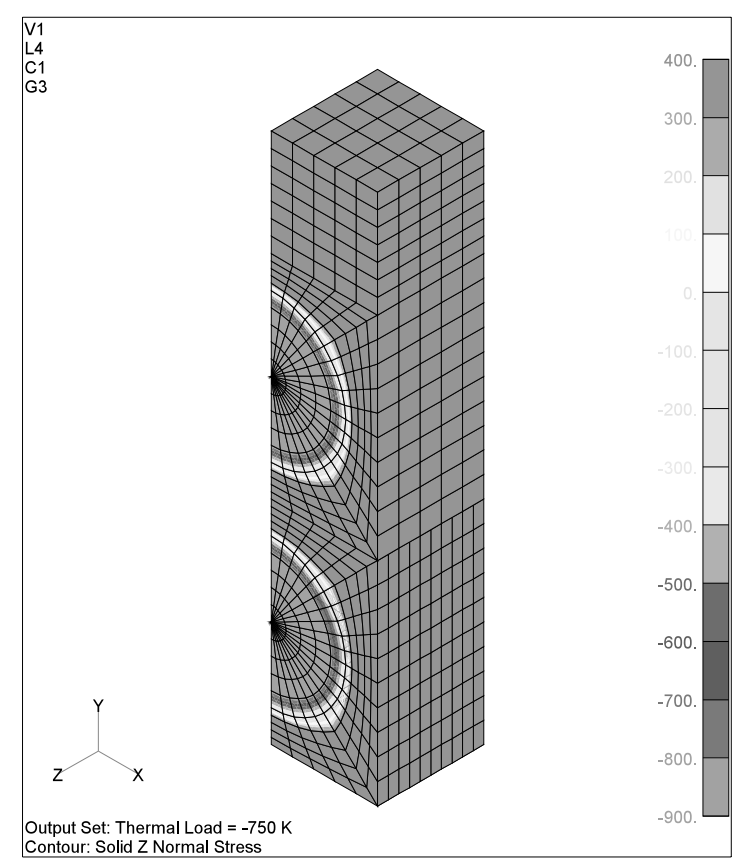

(a)

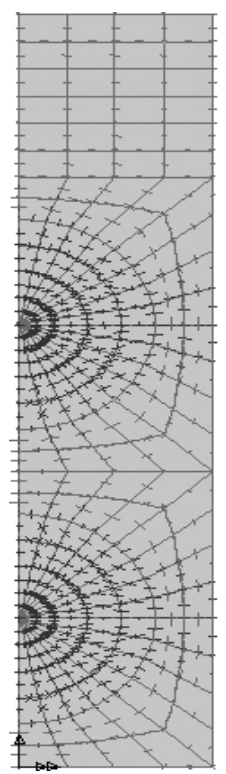

(b)

Fig. 6 Distributions of TRS in symmetric specimen: (a) $\sigma_{z z}$ distribution; (b) Distributions of principle stress in the $X Y$ plane

The $X Y$ Plane $(z=0.1)$ contracted inward as a whole.

Differences are observed in Fig. 5 (a) and (b). In Fig. 5 (a) the fibers are placed symmetrically, the upper section and the lower section of the plate have the same contractions, and therefore no bending deformation is produced. Conversely, in Fig. 5(b), as the fibers are distributed asymmetrically to the neutral axis, the axial thermal stress distribution inside the specimen is asymmetric. We have simulated all five modes in Fig. 4 (b). As the fibers are placed closely to the upper surface of the plate ( $\delta$ value increases), the matrix's tensile stress components in the upper part of the cross section $(y>0.5 \mathrm{~mm})$ increases, and that in the lower part $(y<0.5 \mathrm{~mm})$ of the cross section decreases. This stress imbalance produces a resultant bending moment and causes the specimen to bend. Though not obvious in Fig. 5 (b), the thermal contraction in the upper part of the plate is less than that in the lower part of the plate, and the plate bends upward. Under an identical thermal load $\Delta T=750^{\circ} \mathrm{C}$ the front plane $(z=0.1 \mathrm{~mm})$ of each model rotated an angle $\theta: \theta=\left[u_{z}(\right.$ top $)-u_{z}($ bottom $\left.)\right] / h$. The larger the fiber placement asymmetry is, the larger this angle is. Rotation angles for Model 0 through 4 cases are $0,6.3 \times 10^{-5}$, $1.3 \times 10^{-4}, 1.9 \times 10^{-4}$ and $2.5 \times 10^{-4} \mathrm{rad}$, respectively. These angles are converted to the specimen bending curvature $\kappa$ through the relationship: $\kappa=\theta / d$, resulting the values $\kappa=$ $0,6.35 \times 10^{-4}, 1.3 \times 10^{-3}, 1.9 \times 10^{-3}$ and $2.5 \times 10^{-3} \mathrm{~mm}^{-1}$, respectively. These values are plotted as $\kappa-\Delta$ points in Fig. 3. It is seen that the 3D FEM results compare well with the $1 \mathrm{D}$ beam results. Both results are close to the experimental data.

\section{TRS Effect on Tensile Property}

The thermal-mechanical model, solved either as a 1D beam problem, or as a 3D FEM simulation, gives good predictions of specimen bending. In this section we extend the 3D FEM simulation to evaluate the SiC/Ti composite's in-plane elastic plastic property. In practical experiments it is desirable that tensile specimen be planar without initial bending, only Model 0 with symmetrical fiber placement is analyzed.

Figure 6 plots the triaxial TRS state of the specimen before loading. As Fig. 6(a) shows, the distribution of normal stress in the $Z$-(fiber axial) direction is quite uniform within the fiber or matrix, $\sigma_{m}=395 \mathrm{MPa}$, $\sigma_{f}=-870 \mathrm{MPa}$. However, the principle stresses in the specimen's cross-section are somewhat complicated, as shown in Fig. 6(b). This is attributed to the complex interactions among fibers, matrix, and free surfaces. In general in the cross-sectional directions the fiber is compressed and the matrix is stretched. There are exceptions, however, as in the regions where the fibers are close to each other, the matrix is partly compressed due to the proximity of fiber columns.

In titanium matrix, the tensile residual stress does not exceed the yield limit. Normal TRS component in axial direction, $\sigma_{z}^{r e s}=395 \mathrm{MPa}$ is equivalent to about $0.311 \%$ axial strain. As the first order approximation, we can estimate that if the $\mathrm{SiC} / \mathrm{Ti}$ composite is further stretched in the axial direction, it will yield at the strain value $0.52 \%$ 


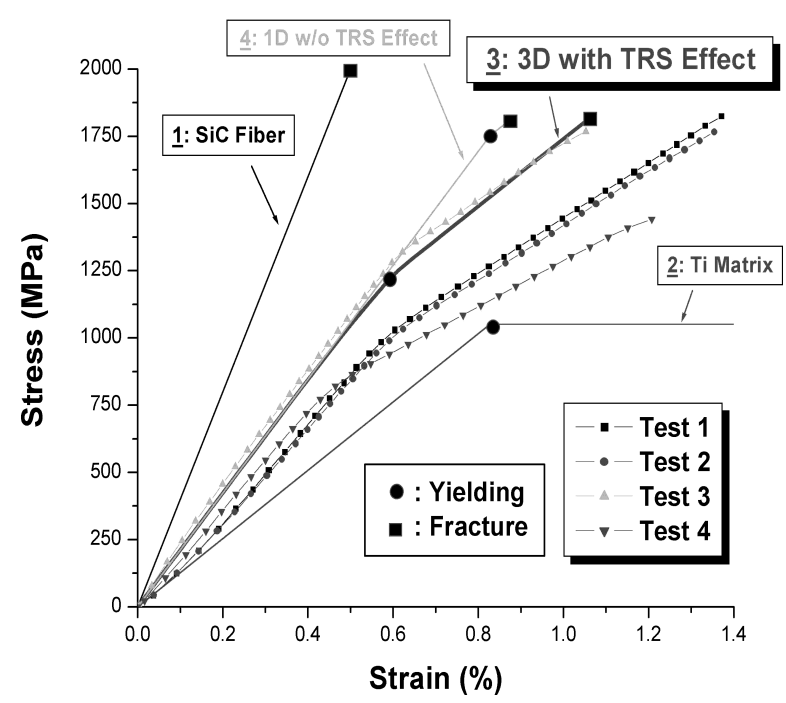

Fig. 7 Stress-strain curves of the SiC fiber, the titanium matrix and the SiC/Ti composites: experimental data and the mechanical predictions

Table 3 Elastic, plastic, and failure parameters of $\mathrm{SiC} / \mathrm{Ti}$ composites: experimental data and predictions by the law of mixture and the FEM simulation

\begin{tabular}{|c|c|c|c|c|}
\hline \multicolumn{2}{|c|}{} & Exp Data & LOM & FEM with TRS \\
\hline \multirow{2}{*}{$E$} & $\mathrm{E}_{\mathrm{L}}(\mathrm{GPa})$ & 203 & 212 & 212 \\
\cline { 2 - 5 } & $v_{\mathrm{LT}}$ & 0.24 & 0.242 & 0.235 \\
\hline \multirow{3}{*}{$\mathbf{P}$} & $\sigma_{\mathrm{y}}(\mathrm{MPa})$ & 1100 & 1750 & 1250 \\
\cline { 2 - 5 } & $\varepsilon_{\mathrm{y}}$ & 0.006 & 0.0083 & 0.0061 \\
\cline { 2 - 5 } & $\mathrm{E}_{\mathrm{P}}(\mathrm{GPa})$ & 112 & 124 & 126 \\
\hline \multirow{2}{*}{$\mathbf{F}$} & $\sigma_{\mathrm{s}}(\mathrm{MPa})$ & 1770 & 1800 & 1800 \\
\cline { 2 - 5 } & $\varepsilon_{\mathrm{s}}$ & 0.0013 & 0.0086 & 0.011 \\
\hline
\end{tabular}

$(=0.83 \%-0.31 \%)$. However, this is not correct because most part of the titanium matrix is under a triaxial tension state. The tensile stress in the transverse directions can be as large as $400 \mathrm{MPa}$. When the composite is pulled in the axial direction, the surrounding tensile stress postpones plastic yielding by stretching the Von-Mises yield surface.

A full 3D evaluation is therefore necessary to calculate the practical elastic-plastic behaviors of the specimen. The analytical model shown in Fig. 4 (b) (Model 0 ) is used here, where both the fiber and the matrix preserve the thermal residual stresses (More precisely said, the thermal strain). With the same constraints described above, a concentrated force load equivalent to $2000 \mathrm{MPa}$ normal stress is applied in Z-direction. The FEM analysis is nonlinear, with the total load $\left(\sigma_{z}=2000 \mathrm{MPa}\right)$ being divided into 40 steps. At each step deformation of the cell is calculated and converted into the normal strain in $Z$-direction. Stress-strain relationship in Z-direction thus simulated is plotted in Fig. 7 (Curve 3). Although some extent of plastic yielding takes place locally (in regions where fibers are close to each other) in the earlier stage, the massive matrix yielding does not happen until a late stage. Curve 3 exhibits an apparent plastic yield stress $\sigma_{y}=1250 \mathrm{MPa}$, yield strain $\varepsilon_{y}=0.61 \%$, both values are lower than that given by simple "law of mixtures" not including TRS effect (1-3), but are higher than the uniaxial estimation including TRS effect. Experimental curves are also shown in Fig. 7 for a comparison. Note that the experimental curves exhibit some degree of scatter. However it is understandable that Curve 3 gives a better result than the simple "law of mixtures" (Curve 4). Some material data simulated by present model is listed in Table 3 .

\section{Conclusions}

Tensile properties of the unidirectional $\mathrm{SiC} / \mathrm{Ti}$ composite are tested. Two phenomena related to thermal residual stress, namely specimen bending and plastic yield point moving, are observed. A thermal-mechanical model considering detailed fiber/matrix structure is proposed for evaluating the thermal deformation and the resulted thermal stress. The model can be solved by using a 1D beam approximate analysis, or through 3D numerical simulations. Both solutions provide consistent results on specimen's thermal bending, which compare well with the experimental measurements. The pertinence of the proposed thermal-mechanical model is therefore verified. We further simulate the elastic-plastic property of the composite. The numerically obtained stress-strain relationship agrees reasonably well with the experimental data, and explains the plastic yield point moving phenomenon. It is notable that the current thermal-mechanical approach provides a good way to predict mechanical behavior of other MMC systems.

\section{References}

( 1 ) Taya, M. and Arsenault, R.J., Metal Matrix Composites, Thermomechanical Behavior, (1989), Pergamon Press.

( 2 ) Aboudi, J., Micromechanical Analysis of Composites by Method of Cells-Update, Applied Mechanics Review, Vol.49 (1996), pp.s83-s91.

( 3 ) Xie, M., Soni, S.R., Hanley, D. and Anto, A., The Nonlinear Behavior of Unidirectional SCS-6/Ti-6Al$4 \mathrm{~V}$ Composite, Proceedings of the American Society for Composites, the 11th Technical Conference, (1996), pp.262-271.

( 4 ) Avila, A.F. and Tamma, K.K., Integrated Micro/Macro Thermal-Elastic-Viscoplastic Analysis of Laminate Metal Matrix Composites, J. Thermal Stress, Vol.21 (1998), pp.897-917.

( 5 ) Banks-Sills, L. and Leiderman, V., Macro-Mechanical Material Model for Fiber Reinforced Metal Matrix Composites, Composites B: Engineering, Vol.30 (1999), pp.443-452.

( 6 ) Sofue, Y., Hashimoto, R., Ogawa, A. and Zhou, F., Tensile Tests of SiC/Ti Composites and SP700 Titanium Alloy, NAL TM-742, (1999).

( 7 ) Sofue, Y., Hashimoto, R., Ogawa, A. and Zhou, F., Tensile Tests of Ti Alloy (SP700) and SiC(SCS- 
6)/Ti(SP700) Composites, Proceedings of 14th Fall Annual Conference of Japan Gas Turbine Society, (1999), pp.117-122.

( 8 ) Zhou, F., Sofue, Y., Ogawa, A. and Hashimoto, R., Thermal Residual Stress and Its Effect on the Tensile Behavior of SiC/Ti Composites, Proceedings of 7th JSME/M\&P Conference, (1999), pp.47-48.

(9) Okazaki, M., Tokiya, N. and Nakatani, N., Stable Crack Growth Process in Unidirectional SiC Fiber Re- inforced Ti-24Al-11Nb Matrix Composite, Journal of Society of Material Science of Japan, Vol.46 (1997), pp.787-794.

(10) Zhou, F., Sofue, Y., Ogawa, A. and Hashimoto, R., On the Thermal Residual Stress in SiC/Ti Composites, Part II Specimen Bending Simulation by Micromechanical Analysis, Proceedings of the 40th Aeroengine/Space Propulsion Symposium, (2000), pp.43-48. 